VoL. 68 (2003) [501-515]

\title{
SYSTEM OF GENERALISED SET-VALUED QUASI-VARIATIONAL-LIKE INEQUALITIES
}

\author{
Jianwen Peng
}

\begin{abstract}
In this paper, we shall introduce a system of generalised set-valued quasi-variationallike inequalities, which generalises and unifies systems of generalised vector variational inequalities, systems of variational inequalities, generalised vector quasi-variationallike inequalities as well as various extensions of the classic variational inequalities in the literature. Some existence results for a solution of a system of generalised set-valued quasi-variational-like inequalities without any monotonity are obtained.
\end{abstract}

\section{INTRODUCTION}

The Vector Variational Inequality in a finite dimensional Euclidean space was introduced in [24] and applications were given. Chen and Cheng [10] studied the vector variational inequality in infinite dimensional space and applied it to vector optimisation problems. Since then, many authors $[9,14,11,12,37,40,41,42,44]$ have intensively studied vector variational inequalities under different assumptions in infinitedimensional spaces. Lee, Kim and Cho [27], Lee, Kim and Lee [28], Lin, Yang and Yao [30], Konnov and Yao [26], Daniilidis and Hadiisavvas [16], Yang and Yao [43], and Oettli and Schlager [33] studied the generalised vector variational inequality and obtained some existence results. Chen and $\mathrm{Li}[13]$ and Lee, Lee and Chang [29] introduced and studied generalised vector quasi-variational inequalities and established some existence theorems. Ansari [2, 3], Ding and Tarafdar [21, 22] and Luo [31] studied generalised vector variational-like inequalities. Ding [19] introduced and studied a class of generalised vector quasi-variational-like inequality problems. Pang [34], Cohen and Chaplais [15], Bianchi [7], and Ansari and Yao [5] considered a system of scalar variational inequalities and Pang showed that the traffic equilibrium problem, the spatial equilibrium problem, the Nash equilibrium, and the general equilibrium programming problem can be modeled as a system of variational inequalities. Ansari, Schaible and Yao [4] considered a system of vector variational inequalities and obtained its existence results. Allevi,

Received 19th June, 2003

Supported by the National Natural Science Foundation of China and Applied Basic Research Foundation of Chongqing.

Copyright Clearance Centre, Inc. Serial-fee code: 0004-9727/03 \$A2.00+0.00. 
Gnudi and Konnov [1] considered a system of generalised vector variational inequalities and established some existence results with relative pseudomonoyonicity.

This paper introduces, a system of generalised set-valued quasi-variational-like inequalities, which generalises and unifies systems of generalised vector variational inequalities, systems of variational inequalities, generalised vector quasi-variational-like inequality as well as various extensions of the classic variational inequalities in the literature. Further some existence results of a solution for system of generalised set-valued quasivariational-like inequalities without any monotonity are proved.

\section{Problem statement and Preliminaries}

Let intA denote the interior of a set $A$ and $I$ be an index set, for each $i \in I$, let $Y_{i}$ be a Hausdorff topological vector space, $E_{i}$ be a locally convex Hausdorff topological vector space. Consider a family of nonempty convex subsets $\left\{X_{i}\right\}_{i \in I}$ with $X_{i} \subset E_{i}$. Let $X=\prod_{i \in I} X_{i}$, and $E=\prod_{i \in I} E_{i}$. An element of the set $X^{i}=\prod_{j \in I \backslash i} X_{i}$ will be denoted by $x^{i}$, therefore, $x \in X$ will be written as $x=\left(x^{i}, x_{i}\right) \in X^{i} \times X_{i}$. For each $i \in I$, let $\eta_{i}: X_{i} \times X_{i} \rightarrow E_{i}$ be a single-valued mapping and $C_{i}: X \rightarrow 2^{Y_{i}}$ be a set-valued mapping such that $C_{i}(x)$ is a closed pointed and convex cone with intC $C_{i}(x) \neq \emptyset$ for each $x \in X$. Let $D_{i}: X \rightarrow 2^{X_{i}}$ and $T_{i}: X \rightarrow 2^{L\left(E_{i}, Y_{i}\right)}$ be two set-valued mappings, where $L\left(E_{i}, Y_{i}\right)$ denotes the space of all continuous linear operators from $E_{i}$ into $Y_{i}$. Then, we consider a system of generalised set-valued quasi-variational-like inequalities, which is to find $\bar{x}=\left(\overline{x^{i}}, \overline{x_{i}}\right)$ in $X$ such that for each $i \in I, \overline{x_{i}} \in D_{i}(\bar{x})$,

$$
\forall y_{i} \in D_{i}(\bar{x}), \exists \overline{v_{i}} \in T_{i}(\bar{x}):\left\langle\overline{v_{i}}, \eta_{i}\left(y_{i}, \overline{x_{i}}\right)\right\rangle \notin-\operatorname{intC} i(\bar{x}) .
$$

Then the point $\bar{x}$ is said to be a solution of the system of generalised set-valued quasivariational-like inequalities.

It is easy to see that $\bar{x}$ is a solution of the system of generalised set-valued quasivariational-like inequalities is equivalent to for each $i \in I$,

$$
\overline{x_{i}} \in D_{i}(\bar{x}), \quad \forall y_{i} \in D_{i}(\bar{x}):\left\langle T_{i}(\bar{x}), \eta_{i}\left(y_{i}, \overline{x_{i}}\right)\right\rangle \notin-\operatorname{int} C_{i}(\bar{x}) .
$$

Where

$$
\left\langle T_{i}(\bar{x}), \eta_{i}\left(y_{i}, \overline{x_{i}}\right)\right\rangle=\bigcup_{v_{i} \in T_{i}(\bar{x})}\left\langle v_{i}, \eta_{i}\left(y_{i}, \overline{x_{i}}\right)\right\rangle .
$$

The following problems are the special cases of the system of generalised set-valued quasi-variational-like inequalities.

(i) For each $i \in I, \eta_{i}\left(y_{i}, x_{i}\right)=y_{i}-x_{i}$ for all $x_{i}, y_{i} \in X_{i}$, then the system of generalised set-valued quasi-variational-like inequalities reduces to the system of generalised set-valued quasi-variational inequalities which is to find $\bar{x}=\left(\overline{x^{i}}, \overline{x_{i}}\right)$ in $X$ such that for each $i \in I, \overline{x_{i}} \in D_{i}(\bar{x})$,

$$
\forall y_{i} \in D_{i}(\bar{x}), \exists \overline{v_{i}} \in T_{i}(\bar{x}):\left\langle\overline{v_{i}}, y_{i}-\overline{x_{i}}\right\rangle \notin-\operatorname{intC}_{i}(\bar{x}) .
$$


(ii) For each $i \in I$, if $D_{i}(x) \equiv X_{i}$ for all $x \in X$, then the system of generalised setvalued quasi-variational-like inequalities reduces to the system of generalised set-valued variational-like inequalities which is to find $\bar{x}=\left(\overline{x^{i}}, \overline{x_{i}}\right)$ in $X$ such that for each $i \in I$,

$$
\forall y_{i} \in X_{i}, \exists \overline{v_{i}} \in T_{i}(\bar{x}):\left\langle\overline{v_{i}}, \eta_{i}\left(y_{i}, x_{i}\right)\right\rangle \notin-\operatorname{intC}_{i}(\bar{x})
$$

(iii) For each $i \in I$, if $D_{i}(x) \equiv X_{i}$ for all $x \in X$, and $\eta_{i}\left(y_{i}, x_{i}\right)=y_{i}-x_{i}$ for all $x_{i}, y_{i} \in X_{i}$, then the system of generalised set-valued quasi-variational-like inequalities reduces to the system of generalised set-valued variational inequalities which is to find $\bar{x}=\left(\overline{x^{i}}, \overline{x_{i}}\right)$ in $X$ such that

$$
\forall y_{i} \in X_{i}, \exists \overline{v_{i}} \in T_{i}(\bar{x}):\left\langle\overline{v_{i}}, y_{i}-\overline{x_{i}}\right\rangle \notin-\operatorname{intC}_{i}(\bar{x})
$$

It is worth noting that the system of generalised set-valued quasi-variational-like inequalities, the system of generalised set-valued quasi-variational inequalities, the system of generalised set-valued variational-like inequalities and the system of generalised setvalued variational inequalities are new models of mathematics.

For each $i \in I$, for all $x \in X$, if $Y_{i} \equiv Y$ and $C_{i}(x) \equiv C$, where $C$ is a convex closed and pointed cone in $Y$ with intC $\neq \emptyset$, then the system of generalised set-valued variational inequalities reduces to a system of set-valued variational inequalities which is to find $\bar{x}=\left(\overline{x^{i}}, \overline{x_{i}}\right)$ in $X$ such that

$$
\forall y_{i} \in X_{i}, \exists \overline{v_{i}} \in T_{i}(\bar{x}):\left\langle\overline{v_{i}}, y_{i}-\overline{x_{i}}\right\rangle \notin-\operatorname{intC} .
$$

This was studied by Allevi, Gnudi and Konnov [1].

If $T_{i}$ is single-valued function, then the system of set-valued variational inequalities reduces to the system of vector variational inequalities, which is to find $\bar{x}=\left(\overline{x^{i}}, \overline{x_{i}}\right)$ in $X$ such that

$$
\left\langle T_{i}(\bar{x}), y_{i}-\overline{x_{i}}\right\rangle \notin-\operatorname{intC}, \quad \forall y_{i} \in X_{i} .
$$

This was considered by Ansari, Schaible and Yao [4].

(iv) For each $i \in I$, for all $x \in X \subseteq R^{n}$, let $Y_{i} \equiv R$ and $C_{i}(x) \equiv R^{+}$ $=\{r \in R: r \geqslant 0\}$, let $T_{i}$ be replaced by $f_{i}: X \rightarrow R$, then the system of vector variational inequalities reduces to the system of scalar variational inequalities which is to find $\bar{x}=\left(\overline{x^{i}}, \overline{x_{i}}\right)$ in $X$ such that

$$
\left\langle f_{i}(\bar{x}), y_{i}-\overline{x_{i}}\right\rangle \geqslant 0, \quad \forall y_{i} \in X_{i}
$$

This was considered in $[34]$ and $[15,7,5]$.

(v) If $I=\{1\}$, then the system of generalised set-valued quasi-variational-like inequalities reduces to the generalised set-valued quasi-variational-like inequality as finding $\bar{x}$ in $X$ such that $\bar{x} \in D(\bar{x})$, and

$$
\forall y \in D(\bar{x}), \exists \widehat{v} \in T(\bar{x}):\langle\widehat{v}, \eta(y, \bar{x})\rangle \notin-\operatorname{intC}(\bar{x}) .
$$


This was introduced and studied by Ding [19] with $C^{+}-\eta$-monotone and weakly $C^{+}-\eta$ monotone condions.

For all $x \in X$, if $D(x) \equiv X$, then the generalised set-valued quasi-variational-like inequality reduces to the generalised set-valued variational-like inequality problem (in short, GSVLI) which is to find $\bar{x}$ in $X$ such that

$$
\forall y \in X, \exists \widehat{v} \in T(\bar{x}):\langle\widehat{v}, \eta(y, \bar{x})\rangle \notin-\operatorname{intC}(\bar{x}) .
$$

This was studied in $[\mathbf{2}, \mathbf{3}, \mathbf{2 1}, \mathbf{2 2}, \mathbf{3 1}]$.

If $T$ is a single-valued mapping and $\eta(y, x)=y-g(x), \quad \forall x, y \in X$, and $D(x) \equiv X$ for all $x \in X$, where $g: X \rightarrow E$ is a single-valued mapping, then the generalised setvalued quasi-variational-like inequality reduces to find $\bar{x}$ in $X$ such that

$$
\langle T(\bar{x}), y-g(\bar{x})\rangle \notin-\operatorname{intC}(\bar{x}), \quad \forall y \in X .
$$

This was considered by Siddiqi, Ansari and Khaliq in [37].

If $\eta(y, x)=y-x$, for all $x, y \in X$, then the generalised set-valued quasi-variationallike inequality reduces to finding $\bar{x}$ in $X$ such that $\bar{x} \in D(\bar{x})$, and

$$
\forall y \in D(\bar{x}), \exists \widehat{v} \in T(\bar{x}):\langle\widehat{v}, y-x\rangle \notin-\operatorname{intC}(\bar{x}) .
$$

This problem was called the generalised set-valued quasi-variational inequality problem, which is new. When $C(x)=C$, for all $x \in X$ is a constant cone, the generalised setvalued quasi-variational problems reduces to the set-valued quasi-variational inequality problem which was studied by Chen and Li [13] and Lee, Lee and Chang [29].

If $D(x) \equiv X$, for all $x \in X$ and $\eta(y, x)=y-x$, for all $x, y \in X$, then the generalised set-valued quasi-variational-like inequality reduces to find $\bar{x}$ in $X$ such that

$$
\forall y \in X, \exists \widehat{v} \in T(\bar{x}):\langle\widehat{v}, \eta(y, \bar{x})\rangle \notin-\operatorname{intC}(\bar{x}) .
$$

This Problem and its special cases are called the generalised vector variational inequality which was introduced and studied in $[27,28,30,26,16,43,33]$.

If $T$ is single-valued function and $D(x) \equiv X$, for all $x \in X$, then the generalised set-valued quasi-variational-like inequality reduces to find $\bar{x}$ in $X$ such that

$$
\langle T(\bar{x}), \eta(y, \bar{x})\rangle \notin-\operatorname{intC}(\bar{x}), \quad \forall y \in X .
$$

This problem and its special cases were studied by many authors, see $[27,9,14,11$, $12,37,40,41,42,44]$.

If $Y=R$ and $C(x)=[0, \infty)$, for all $x \in X$, then $L(E, Y)=E^{*}$, where $E^{*}$ is the dual space of $E$, and the generalised set-valued quasi-variational-like inequality reduces to find $\bar{x}$ in $X$ such that $\bar{x} \in D(\bar{x})$, and

$$
\forall y \in D(\bar{x}), \exists \widehat{v} \in T(\bar{x}):\langle\widehat{v}, \eta(y, \bar{x})\rangle \geqslant 0 .
$$


This problem includes many classes of scalar type generalised quasi-variational inequality and generalised quasi-variational-like inequality problems as special cases, see $[\mathbf{3 6}, 46$, $8,20,17,18,45]$.

In order to prove the main results, we need the following definitions and lemmas.

Definition 2.1: For each $i \in I$, let $E_{i}, Y_{i}$ be two real topological vector space, $X_{i}$ be a nonempty and convex subset of $E_{i}, C_{i}: X \rightarrow 2^{Y_{i}}$ be a set-valued mapping such that $C_{i}(x)$ is a closed pointed and convex cone for each $x \in X$. Let $\eta_{i}: X_{i} \times X_{i} \rightarrow E_{i}$ be a single-valued mapping. $T_{i}: X \rightarrow 2^{L\left(E_{i}, Y_{i}\right)}$ is said to satisfy the generalised partial $L-\eta_{i}$-condition if and only if for any finite set $\left\{y_{i_{1}}, y_{i_{2}}, \ldots, y_{i_{n}}\right\}$ in $X_{i}$, for all $\bar{x}=\left(\overline{x^{i}}, \overline{x_{i}}\right)$ with $\overline{x_{i}}=\sum_{j=1}^{n} \alpha_{j} y_{i_{j}}$, where $\alpha_{j} \geqslant 0$ and $\sum_{j=1}^{n} \alpha_{j}=1$, there exists $\overline{v_{i}} \in T_{i}(\bar{x})$ such that

$$
\left\langle\overline{v_{i}}, \sum_{j=1}^{n} \alpha_{j} \eta_{i}\left(y_{i_{j}}, \overline{x_{i}}\right)\right\rangle \notin-\operatorname{intC}_{i}(\bar{x})
$$

REMARK 2.1. If $I=\{1\}$, then Definition 2.1 reduces to the generalised $L-\eta$-condition in $[21]$.

REMARK 2.2. If $\eta_{i}\left(y_{i}, x_{i}\right)$ is affine in the first argument and for all $x=\left(x^{i}, x_{i}\right) \in X$, $\exists \overline{v_{i}} \in T_{i}(x)$, such that

$$
\left\langle\bar{v}_{i}, \eta_{i}\left(x_{i}, x_{i}\right)\right\rangle \notin-\operatorname{int}_{i}(x) .
$$

Then $T_{i}$ satisfy the generalised partial $L-\eta_{i}$-condition.

REMARK 2.3. If $\eta_{i}\left(y_{i}, x_{i}\right)=y_{i}-x_{i}$, for all $x_{i}, y_{i} \in X_{i}$, then for any finite set $\left\{y_{i_{1}}, y_{i_{2}}, \ldots, y_{i_{n}}\right\}$ in $X_{i}$, for all $\bar{x}=\left(\overline{x^{i}}, \overline{x_{i}}\right)$ with $\overline{x_{i}}=\sum_{j=1}^{n} \alpha_{j} y_{i_{j}}$, where $\alpha_{j} \geqslant 0$ and $\sum_{j=1}^{n} \alpha_{j}=1$, we have that

$$
\left.\left\langle\bar{v}, \sum_{j=1}^{n} \alpha_{j}\left(y_{i_{j}}-\overline{x_{i}}\right)\right\rangle=\left\langle\bar{v}, \overline{x_{i}}-\overline{x_{i}}\right)\right\rangle=0 \notin-\operatorname{intC}_{i}(\bar{x}), \quad \forall \bar{v} \in T_{i}(\bar{x}) .
$$

And hence $T_{i}$ satisfy the generalised partial $L-\eta_{i}$-condition trivially.

Definition 2.2: ([6].) Let $X$ and $Y$ be two topological spaces and $T: X \rightarrow 2^{Y}$ be a set-valued mapping. Then

(1) $T$ is said to be upper semicontinuous if, for any $x_{0} \in X$ and for each open set $U$ in $Y$ containing $T\left(x_{0}\right)$, there is a nerghborhood $V$ of $x_{0}$ in $X$ such that $T(x) \subseteq U$, for all $x \in V$.

(2) $T$ is said to have open lower sections if the set $T^{-1}(y)=\{x \in X: y \in T(x)\}$ is open in $X$ for each $y \in Y$.

(3) $T$ is said to be closed, if the set $\{(x, y) \in X \times Y: y \in T(x)\}$ is closed in $X \times Y$. 
Lemma 2.1. ([39].) Let $X$ be a paracompact Hausdorff space and $Y$ be a linear topological space. Suppose $T: X \rightarrow 2^{Y}$ is a set-valued mapping such that

(i) for each $x \in X, T(x)$ is nonempty,

(ii) for each $x \in X, T(x)$ is convex, and

(iii) $T$ has open lower sections. Then there exists a continuous function $f$ : $X \rightarrow Y$ such that $f(x) \in T(x)$ for all $x \in X$.

Lemma 2.2. ([6].) Let $X$ and $Y$ be topological spaces. If $T: X \rightarrow 2^{Y}$ is an upper semicontinuous set-valued mapping with closed values, then $T$ is closed.

Lemma 2.3. ([38].) Let $X$ and $Y$ be topological spaces and $T: X \rightarrow 2^{Y}$ is an upper semicontinuous set-valued mapping with compact values. Suppose $\left\{x_{\alpha}\right\}$ is a net in $X$ such that $x_{\alpha} \rightarrow x_{0}$. If $y_{\alpha} \in T\left(x_{\alpha}\right)$ for each $\alpha$, then there is a $y_{0} \in T\left(x_{0}\right)$ and a subset $\left\{y_{\beta}\right\}$ of $\left\{y_{\alpha}\right\}$ such that $y_{\beta} \rightarrow y_{0}$.

Lemma 2.4. ([39].) Let $X$ and $Y$ be two topological spaces. Suppose $T: X$ $\rightarrow 2^{Y}$ and $K: X \rightarrow 2^{Y}$ are set-valued mappings having open lower sections, then

(i) The set-valued mapping $F: X \rightarrow 2^{Y}$ defined by, for each $x \in X, F(x)$ $=\mathrm{Co}(T(x))$ has open lower sections.

(ii) The set-valued mapping $\theta: X \rightarrow 2^{Y}$ defined by, for each $x \in X, \theta(x)$ $=T(x) \cap K(x)$ has open lower sections.

LEMma 2.5. ([23].) Let $E$ be a locally convex topological linear space and $X$ be a compact convex subset in $E$. Suppose $T: X \rightarrow 2^{X}$ is a set-valued mapping such that

(i) for each $x \in X, T(x)$ is nonempty,

(ii) for each $x \in X, T(x)$ is convex and closed,

(iii) $T$ is upper semicontinuous.

Then there exists a $\bar{x} \in X$ such that $\bar{x} \in T(\bar{x})$.

Let $Y$ be a real Hausdorff topological vector space and $X$ be a nonempty convex subsets in a real locally convex Hausdorff topological vector space $E$. We denote by $L(E, Y)$ the space of all continuous linear operators from $E$ into $Y$ and by $\langle u, y\rangle$ the evaluation of $u \in L(E, Y)$ at $y \in E$. Let $\sigma$ be the family of all bounded subsets of $X$ whose union is total in $E$, that is, the linear hull of $\cup\{S: S \in \sigma\}$ is dense in $X$. Let $\beta$ be a neighbourhood base of 0 in $Y$. When $S$ runs through $\sigma, V$ through $\beta$, the family

$$
M(S, V)=\left\{l \in L(E, Y): \bigcup_{x \in S}\langle l, x\rangle \subset V\right\}
$$

is a neighbourhood base of 0 in $L(E, Y)$ at $x \in E$ (see [35, pp. 79-80]). By the Corollary of Schaefer [35, pp. 80], $L(E, Y)$ becomes a locally convex topological vector space under the $\sigma$-topology, where $Y$ is assumed a locally convex topological space. 
Lemma 2.6. ([21, 19].) Let $E$ and $Y$ be real Hausdorff topological vector spaces and $L(E, Y)$ be the topological vector space under the $\sigma$-topology. Then, the bilinear mapping

$$
\langle., .\rangle: L(E, Y) \times E \rightarrow Y
$$

is continuous on $L(E, Y)$, where $\langle l, x\rangle$ denotes the evaluation of the linear operator $l$ $\in L(X, Y)$ at $x \in X$.

\section{EXISTENCE RESULTS}

In this section, we shall present some existence results for a solution to the system of generalised set-valued quasi-variational-like inequalities without any monotone conditions.

THEOREM 3.1. Let $I$ be an index set and $I$ be countable. For each $i \in I$, let $Y_{i}$ be a real Hausdorff topological vector space, $X_{i}$ be a nonempty, compact, convex and metrisable set in a real locally convex Hausdorff topological vector space $E_{i}$, let $D_{i}: X \rightarrow 2^{X_{i}}$ be an upper semicontinuous set-valued mapping with nonempty convex closed values and open lower sections, and let $L\left(E_{i}, Y_{i}\right)$ be equipped with the $\sigma$-topology. Suppose that

(i) for each $i \in I, C_{i}: X \rightarrow 2^{Y_{i}}$ is a set-valued mapping such that $C_{i}(x)$ is a closed pointed and convex cone with int $C_{i}(x) \neq \emptyset$ for each $x \in X$, and the set-valued mapping $M_{i}=Y_{i} \backslash\left(-\right.$ intC $\left._{i}\right): X \rightarrow 2^{Y_{i}}$ be upper semicontinuous;

(ii) for each $i \in I, T_{i}: X \rightarrow 2^{L\left(E_{i}, Y_{i}\right)}$ is an upper semicontinous set-valued mapping with nonempty compact values, $\eta_{i}: X_{i} \times X_{i} \longrightarrow E_{i}$ be continuous with respect to the second argument, such that $T_{i}$ satisfies the generalised partial $L-\eta_{i}$-condition.

Then, there exists $\bar{x}=\left(\overline{x^{i}}, \overline{x_{i}}\right)$ in $X$ such that for each $i \in I, \overline{x_{i}} \in D_{i}(\bar{x})$ and for all $y_{i} \in D_{i}(\bar{x}), \exists \overline{v_{i}} \in T_{i}(\bar{x}):\left\langle\overline{v_{i}}, \eta_{i}\left(y_{i}, \overline{x_{i}}\right)\right\rangle \notin-\operatorname{intC}_{i}(\bar{x})$. that is, the system of generalised set-valued quasi-variational-like inequalities has a solution $\bar{x} \in X$.

Proof: For each $i \in I$, define a set-valued mapping $P_{i}: X \rightarrow 2^{X_{i}}$ by

$$
\begin{aligned}
P_{i}(x) & =\left\{y_{i} \in X_{i}:\left\langle T_{i}(x), \eta_{i}\left(y_{i}, x_{i}\right)\right\rangle \subseteq-\operatorname{intC}_{i}(x)\right\} \\
& =\left\{y_{i} \in X_{i}:\left\langle v_{i}, \eta_{i}\left(y_{i}, x_{i}\right)\right\rangle \in-\operatorname{intC}_{i}(x), \quad \forall v_{i} \in T_{i}(x)\right\}, \quad \forall x \in X .
\end{aligned}
$$

Thus, proving the theorem is equivalent to showing that there exists $\bar{x} \in X$ such that, for each $i \in I, \overline{x_{i}} \in D_{i}(\bar{x})$ and $D_{i}(\bar{x}) \cap P_{i}(\bar{x})=\emptyset$.

We first prove that $x_{i} \notin \operatorname{Co}\left(P_{i}(x)\right)$ for all $x=\left(x^{i}, x_{i}\right) \in X$. To see this, suppose, by way of contradiction, that there exists some $i \in I$ and some point $\bar{x}=\left(\overline{x^{i}}, \overline{x_{i}}\right) \in X$ such that $\overline{x_{i}} \in \operatorname{Co}\left(P_{i}(\bar{x})\right)$. Then there exists a finite number of points $y_{i_{1}}, y_{i_{2}}, \ldots, y_{i_{n}}$ in $X_{i}$, and $\alpha_{j} \geqslant 0$ with $\sum_{j=1}^{n} \alpha_{j}=1$ such that $\bar{x}=\sum_{j=1}^{n} \alpha_{j} y_{i_{j}}$ and $y_{i_{j}} \in P(\bar{x})$ for all $j=1,2, \ldots, n$. 
That is,

$$
\left\langle v_{i}, \eta_{i}\left(y_{i}, \overline{x_{i}}\right)\right\rangle \in-\operatorname{intC}_{i}(\bar{x}), \quad \forall v_{i} \in T_{i}(\bar{x})
$$

and $j=1,2, \ldots, n$. Since $\operatorname{int} C_{i}(\bar{x})$ is convex, we obtain

$$
\left\langle v_{i}, \sum_{j=1}^{n} \alpha_{j} \eta_{i}\left(y_{i}, \overline{x_{i}}\right)\right\rangle \in-\operatorname{intC}_{i}(\bar{x}), \quad \forall v_{i} \in T_{i}(x),
$$

which contradicts the fact that $T_{i}$ satisfies the generalised partial $L-\eta_{i}$-condition. Therefore $x_{i} \notin \mathrm{Co}\left(P_{i}(x)\right)$ for all $x \in X$.

Now we prove that the set

$$
\begin{aligned}
P_{i}^{-1}\left(y_{i}\right) & =\left\{x \in X:\left\langle T_{i}(x), \eta_{i}\left(y_{i}, x_{i}\right)\right\rangle \subseteq-\operatorname{intC}_{i}(x)\right\} \\
& =\left\{x \in X:\left\langle v_{i}, \eta_{i}\left(y_{i}, x_{i}\right)\right\rangle \in-\operatorname{intC}_{i}(x), \quad \forall v_{i} \in T_{i}(x)\right\}
\end{aligned}
$$

is open for each $i \in I$ and for each $y_{i} \in X_{i}$. That is, $P_{i}$ has open lower sections in $X$. We only need to prove that

$$
\begin{aligned}
S_{i}\left(y_{i}\right) & =\left\{x \in X:\left\langle T_{i}(x), \eta_{i}\left(y_{i}, x_{i}\right)\right\rangle \notin-\operatorname{intC}_{i}(x)\right\} \\
& =X \backslash P_{i}^{-1}\left(y_{i}\right)=\left\{x \in X: \exists v_{i} \in T_{i}(x) \text { such that }\left\langle v_{i}, \eta_{i}\left(y_{i}, x_{i}\right)\right\rangle \notin-\operatorname{intC}_{i}(x)\right\} .
\end{aligned}
$$

is closed for all $y_{i} \in X_{i}$.

In fact, consider a net $x_{t} \in S_{i}\left(y_{i}\right)$ such that $x_{t} \rightarrow x \in X$. Since $x_{t} \in S_{i}\left(y_{i}\right)$, there exists $s_{t} \in T_{i}\left(x_{t}\right)$ such that

$$
\left\langle s_{t}, \eta_{i}\left(y_{i}, x_{i_{t}}\right)\right\rangle \notin-\operatorname{intC}{ }_{i}\left(x_{t}\right) .
$$

From the upper semicontinuous and compact values of $T_{i}$ and Lemma 2.3, it suffices to find a subset $\left\{s_{t_{j}}\right\}$ which converges to some $s \in T_{i}(x)$. By Lemma 2.6, we know that (.) is continuous, and hence

$$
\left\langle s_{t_{j}}, \eta_{i}\left(y_{i}, x_{i_{t_{j}}}\right)\right\rangle \rightarrow\left\langle s, \eta_{i}\left(y_{i}, x_{i}\right)\right\rangle .
$$

By Lemma 2.2 and upper semicontinuity of $M_{i}$, we have $\left\langle s, \eta_{i}\left(y_{i}, x_{i}\right)\right\rangle \notin-\operatorname{intC}_{i}(x)$, and hence $x \in S_{i}\left(y_{i}\right), S_{i}\left(y_{i}\right)$ is closed. For each $i \in I$, also define another set-valued mapping, $G_{i}: X \rightarrow 2^{X_{i}}$ by $G_{i}(x)=D_{i}(x) \cap \mathrm{Co}\left(P_{i}(x)\right)$, for all $x \in X$. Let the set $W_{i}=\left\{x \in X: G_{i}(x) \neq \emptyset\right\}$. Since $D_{i}$ and $P_{i}$ has open lower sections in $X$, and by Lemma 2.4, we know that $\operatorname{Co}\left(P_{i}\right)$ and $G_{i}$ also has open lower sections in $X$. Hence, $W_{i}=\bigcup_{y_{i} \in X_{i}} G_{i}^{-1}\left(y_{i}\right)$ is an open set in $X$. Then, the set-valued mapping $\left.G_{i}\right|_{W_{i}}: W_{i} \rightarrow 2^{X_{i}}$ has open lower sections in $W_{i}$, and for all $x \in W_{i}, G_{i}(x)$ is nonempty and convex. Also, since $X$ is a metrisable space $[25$, p. 50$], W_{i}$ is paracompact $[32$, p .831]. Hence, by 
Lemma 2.1, there is a continuous function $f_{i}: W_{i} \rightarrow X_{i}$ such that $f_{i}(x) \in G_{i}(x) \subset D_{i}(x)$ for all $x \in W_{i}$. Define $T_{i}: X \rightarrow 2^{X_{i}}$ by

$$
T_{i}(x)= \begin{cases}f_{i}(x) & \text { if } x \in W_{i}, \\ D_{i}(x) & \text { if } x \notin W_{i} .\end{cases}
$$

Now, we prove that $T_{i}$ is upper semicontinuous. In fact, for each open set $V_{i}$ in $X_{i}$, the set

$$
\begin{aligned}
\left\{x \in X: T_{i}(x) \subset V_{i}\right\}=\left\{x \in W_{i}:\right. & \left.f_{i}(x) \in V_{i}\right\} \cup\left\{x \in X \backslash W_{i}: D_{i}(x) \subset V_{i}\right\} \\
& \subset\left\{x \in W_{i}: f_{i}(x) \in V_{i}\right\} \cup\left\{x \in X: D_{i}(x) \subset V_{i}\right\} .
\end{aligned}
$$

On the other hand, when $x \in W_{i}$, and $f_{i}(x) \in V_{i}$, we have $T_{i}(x)=f_{i}(x) \in V_{i}$. when $x \in X$ and $D_{i}(x) \subset V_{i}$, since $f_{i}(x) \in D_{i}(x)$ if $x \in W_{i}$, we know that $T_{i}(x) \subset V_{i}$ and so

$$
\left\{x \in W_{i}: f_{i}(x) \in V_{i}\right\} \cup\left\{x \in X: D_{i}(x) \subset V_{i}\right\} \subset\left\{x \in X: T_{i}(x) \subset V_{i}\right\} .
$$

Therefore,

$$
\left\{x \in X: T_{i}(x) \subset V_{i}\right\}=\left\{x \in W_{i}: f_{i}(x) \in V_{i}\right\} \cup\left\{x \in X: D_{i}(x) \subset V_{i}\right\}
$$

Since $f_{i}$ is continuous and $D_{i}$ is upper semicontinuous, the sets $\left\{x \in W_{i}: f_{i}(x) \in V_{i}\right\}$ and $\left\{x \in X: D_{i}(x) \subset V_{i}\right\}$ are open. It follows that $\left\{x \in X: T_{i}(x) \subset V_{i}\right\}$ is open and so the mapping $T_{i}: X \rightarrow 2^{X_{i}}$ is upper semicontinuous. Now define $T: X \rightarrow 2^{X}$ by $T(x)=\prod_{i \in I} T_{i}(x)$, for each $x \in X$. By Lemma 3 [23, p .124], $T$ is upper semicontinuous. Since for each $x \in X, T(x)$ is convex, closed, and nonempty, by Lemma 2.5, there is $\bar{x} \in X$ such that $\bar{x} \in T(\bar{x})$. Note that for each $i \in I, \bar{x} \notin W_{i}$. Otherwise, there is some $i \in I$ such that $\bar{x} \in W_{i}$. then $\overline{x_{i}}=f_{i}(\bar{x}) \in \operatorname{Co}\left(P_{i}(\bar{x})\right)$, which contradicts $x_{i} \in \operatorname{Co}\left(P_{i}(x)\right)$ for all $x=\left(x^{i}, x_{i}\right) \in X$. Thus $\overline{x_{i}} \in D_{i}(\bar{x})$ and $G_{i}(\bar{x})=\emptyset$ for each $i \in I$. That is, $\overline{x_{i}} \in D_{i}(\bar{x})$ and $D_{i}(\bar{x}) \cap \operatorname{Co}\left(P_{i}(\bar{x})\right)=\emptyset$ for each $i \in I$, which implies $\overline{x_{i}} \in D_{i}(\bar{x})$ and $D_{i}(\bar{x}) \cap P_{i}(\bar{x})=\emptyset$ for each $i \in I$. Consequently, there exists $\bar{x}=\left(\overline{x^{i}}, \overline{x_{i}}\right)$ in $X$ such that for each $i \in I$,

$$
\overline{x_{i}} \in D_{i}(\bar{x}) \text { and } \quad \forall y_{i} \in D_{i}(\bar{x}), \quad \exists \overline{v_{i}} \in T_{i}(\bar{x}):\left\langle\overline{v_{i}}, \eta_{i}\left(y_{i}, \overline{x_{i}}\right)\right\rangle \notin-\operatorname{intC}_{i}(\bar{x}) .
$$

Hence, the solution set of system of generalised set-valued quasi-variational-like inequalities is nonempty.

By Theorem 3.1 and Remark 2.2, we have

Corollary 3.2. Let $I$ be an index set and $I$ be countable. For each $i \in I$, let $Y_{i}$ be a real Hausdorff topological vector space, $X_{i}$ be a nonempty, compact, convex and metrisable set in a real locally convex Hausdorff topological vector space $E_{i}$, let $D_{i}: X \rightarrow 2^{X_{i}}$ be an upper semicontinuous set-valued mapping with nonempty convex 
closed values and open lower sections, and $L\left(E_{i}, Y_{i}\right)$ be equipped with the $\sigma$-topology. Suppose that

(i) for each $i \in I, C_{i}: X \rightarrow 2^{Y_{i}}$ is a set-valued mapping such that $C_{i}(x)$ is a closed pointed and convex cone with intC $C_{i}(x) \neq \emptyset$ for each $x \in X$, and the set-valued mapping $M_{i}=Y_{i} \backslash\left(-\right.$ intC $\left._{i}\right): X \rightarrow 2^{Y_{i}}$ be upper semicontinuous;

(ii) for each $i \in I, T_{i}: X \rightarrow 2^{L\left(E_{i}, Y_{i}\right)}$ is an upper semicontinuous set-valued mapping with nonempty compact values, $\eta_{i}: X_{i} \times X_{i} \longrightarrow E_{i}$ be continuous with respect to the second argument and affine with respect to the first argument and for all $x=\left(x^{i}, x_{i}\right) \in X, \exists \overline{v_{i}} \in T_{i}(x)$, such that

$$
\left\langle\overline{v_{i}}, \eta_{i}\left(x_{i}, x_{i}\right)\right\rangle \notin-\operatorname{intC}_{i}(x)
$$

Then, there exists $\bar{x}=\left(\overline{x^{i}}, \overline{x_{i}}\right)$ in $X$ such that for each $i \in I$,

$$
\overline{x_{i}} \in D_{i}(\bar{x}) \text { and } \quad \forall y_{i} \in D_{i}(\bar{x}), \exists \overline{v_{i}} \in T_{i}(\bar{x}): \quad\left\langle\overline{v_{i}}, \eta_{i}\left(y_{i}, \overline{x_{i}}\right)\right\rangle \notin-\operatorname{intC}_{i}(\bar{x}) .
$$

that is, the system of generalised set-valued quasi-variational-like inequalities has a solution $\bar{x} \in X$.

If $\eta_{i}\left(y_{i}, x_{i}\right)=y_{i}-x_{i}$, for all $x_{i}, y_{i} \in X_{i}$, by Remark 2.3 and Theorem 3.1, it is easy to obtain the existence of a solution for the system of generalised set-valued quasi-variational inequalities as follows.

CoRollary 3.3. Let $I$ be an index set and $I$ be countable. For each $i \in I$, let $Y_{i}$ be a real Hausdorff topological vector space, $X_{i}$ be a nonempty, compact, convex and metrisable set in a real locally convex Hausdorff topological vector space $E_{i}$, let $D_{i}: X \rightarrow 2^{X_{i}}$ be an upper semicontinuous set-valued mapping with nonempty convex closed values and open lower sections, and $L\left(E_{i}, Y_{i}\right)$ be equipped with the $\sigma$-topology. Suppose that

(i) for each $i \in I, C_{i}: X \rightarrow 2^{Y_{i}}$ is a set-valued mapping such that $C_{i}(x)$ is a closed pointed and convex cone with $\operatorname{int}_{i}(x) \neq \emptyset$ for each $x \in X$, and the set-valued mapping $M_{i}=Y_{i} \backslash\left(-\mathrm{intC}_{i}\right): X \rightarrow 2^{Y_{i}}$ be upper semicontinuous;

(ii) for each $i \in I, T_{i}: X \rightarrow 2^{L\left(E_{i}, Y_{i}\right)}$ is an upper semicontinuous set-valued mapping with nonempty compact values.

Then, there exists $\bar{x}=\left(\overline{x^{i}}, \overline{x_{i}}\right)$ in $X$ such that for each $i \in I$,

$$
\overline{x_{i}} \in D_{i}(\bar{x}) \text { and } \forall y_{i} \in D_{i}(\bar{x}), \exists \overline{v_{i}} \in T_{i}(\bar{x}):\left\langle\overline{v_{i}}, y_{i}-\overline{x_{i}}\right\rangle \notin-\operatorname{intC}_{i}(\bar{x}) .
$$

that is, the system of generalised set-valued quasi-variational inequalities has a solution $\bar{x} \in X$.

REMARK 3.1. If $D_{i}(x)=X_{i}$ and $C_{i}(x)=C$ for each $i \in I$ and for all $x \in X$, where $C$ is a pointed convex cone with intC $\neq \emptyset$, then by Corollary 3.3 , we can obtain the existence 
of a solution for a system of set-valued variational inequalities which is different from those results in [1]. Moreover, let $T_{i}$ be a single-valued mapping, then by Corollary 3.3, we can recover Theorem 3.1 in [4] with the additional condition of metrisablity of $X_{i}$. Hence, Theorem 3.1, Corollary 3.2 and Corollary 3.3 are generalisations of $[4$, Theorem 3.1].

THEOREM 3.4. Let $I$ be an index set and $I$ be countable. For each $i \in I$, let $Y_{i}$ be a real Hausdorff topological vector space, $X_{i}$ be a nonempty, compact, convex and metrisable set in a real locally convex Hausdorff topological vector space $E_{i}$, let $D_{i}: X \rightarrow 2^{X_{i}}$ be an upper semicontinuous set-valued mapping with nonempty convex closed values and open lower sections, and let $L\left(E_{i}, Y_{i}\right)$ be equipped with the $\sigma$-topology. Suppose that

(i) for each $i \in I, C_{i}: X \rightarrow 2^{Y_{i}}$ is a set-valued mapping such that $C_{i}(x)$ is a closed pointed and convex cone with $\operatorname{int} C_{i}(x) \neq \emptyset$ for each $x \in X$, and the set-valued mapping $M_{i}=Y_{i} \backslash\left(-\right.$ intC $\left._{i}\right): X \rightarrow 2^{Y_{i}}$ be upper semicontinuous;

(ii) for each $i \in I, T_{i}: X \rightarrow 2^{L\left(E_{i}, Y_{i}\right)}$ is an upper semicontinuous set-valued mapping with nonempty compact values, $\eta_{i}: X_{i} \times X_{i} \longrightarrow E_{i}$ be continuous with respect to the second argument;

(iii) for each $i \in I$, there exists a mapping $h_{i}: X_{i} \times X_{i} \rightarrow Y_{i}$, such that:

(a) For all $x=\left(x^{i}, x_{i}\right) \in X, \forall y_{i} \in X_{i}, \exists v_{i} \in T_{i}(x)$, such that

$$
h_{i}\left(x_{i}, y_{i}\right)-\left\langle v_{i}, \eta_{i}\left(y_{i}, x_{i}\right)\right\rangle \in-\operatorname{intC}(x)
$$

(b) For any finite set $\left\{y_{i_{1}}, y_{i_{2}}, \ldots, y_{i_{n}}\right\} \subseteq X_{i}$ and for all $x=\left(x^{i}, x_{i}\right)$ $\in X$ with $x_{i}=\sum_{j=1}^{n} \alpha_{j} y_{i_{j}}$, where $\alpha_{j} \geqslant 0$ and $\sum_{j=1}^{n} \alpha_{j}=1$, there is a $j$ $\in\{1,2, \ldots, n\}$, such that $h_{i}\left(x_{i}, y_{i_{j}}\right) \notin-\operatorname{intC}_{i}(x)$.

Then, there exists $\bar{x}=\left(\overline{x^{i}}, \overline{x_{i}}\right)$ in $X$ such that for each $i \in I$,

$$
\overline{x_{i}} \in D_{i}(\bar{x}) \text { and } \quad \forall y_{i} \in D_{i}(\bar{x}), \exists \overline{v_{i}} \in T_{i}(\bar{x}):\left\langle\overline{v_{i}}, \eta_{i}\left(y_{i}, \overline{x_{i}}\right)\right\rangle \notin-\operatorname{intC}_{i}(\bar{x}) .
$$

that is, the system of generalised set-valued quasi-variational-like inequalities has a solution $\bar{x} \in X$.

Proof: For each $i \in I$, define two set-valued mappings $P_{i}: X \rightarrow 2^{X_{i}}, Q_{i}: X \rightarrow 2^{X_{i}}$ by

$$
\begin{array}{ll}
P_{i}(x) & =\left\{y_{i} \in X_{i}:\left\langle v_{i}, \eta_{i}\left(y_{i}, x_{i}\right)\right\rangle \in-\operatorname{intC}_{i}(x), \quad \forall v_{i} \in T_{i}(x)\right\}, \quad \forall x \in X \\
Q_{i}(x) & =\left\{y_{i} \in X_{i}: h_{i}\left(x_{i}, y_{i}\right) \in-\operatorname{int} C_{i}(x)\right\}, \quad \forall x \in X .
\end{array}
$$

We first prove that $x_{i} \notin \operatorname{Co}\left(Q_{i}(x)\right)$ for each $i \in I$ and for all $x=\left(x^{i}, x_{i}\right) \in X$. To see this, suppose, by way of contradiction, that there exists some point $\bar{x}=\left(\overline{x^{i}}, \overline{x_{i}}\right) \in X$ 
such that $\overline{x_{i}} \in \operatorname{Co}\left(Q_{i}(\bar{x})\right)$. Then there exists finite points $y_{i_{1}}, y_{i_{2}}, \ldots, y_{i_{n}}$ in $X_{i}$, and $\alpha_{j} \geqslant 0$ with $\sum_{j=1}^{n} \alpha_{j}=1$ such that $\overline{x_{i}}=\sum_{j=1}^{n} \alpha_{j} y_{i_{j}}$ and $y_{i_{j}} \in Q_{i}(\bar{x})$ for all $j=1,2, \ldots, n$. That is, $h_{i}\left(\overline{x_{i}}, y_{i_{j}}\right) \in-\operatorname{intC}_{i}(\bar{x}), j=1,2, \ldots, n$. This contradicts the condition (b) of (iii). Therefore $x_{i} \notin \operatorname{Co}\left(Q_{i}(x)\right)$ for each $i \in I$ and for all $x=\left(x^{i}, x_{i}\right) \in X$. The condition (a) of (iii) implies that $Q_{i}(x) \supseteq P_{i}(x)$ for all $x \in X$. Hence, $x_{i} \notin \operatorname{Co}\left(P_{i}(x)\right)$, for all $x=\left(x^{i}, x_{i}\right) \in X$. The remainder of the proof is same as that in the proof of Theorem 3.1.

Corollary 3.5. Let $I$ be an index set and $I$ be countable. For each $i \in I$, let $Y_{i}$ be a real Hausdorff topological vector space, $X_{i}$ be a nonempty, compact, convex and metrisable set in a real locally convex Hausdorff topological vector space $E_{i}$, let $D_{i}: X \rightarrow 2^{X_{i}}$ be an upper semicontinuous set-valued mapping with nonempty convex closed values and open lower sections, and let $L\left(E_{i}, Y_{i}\right)$ be equipped with the $\sigma$-topology. Suppose that

(i) for each $i \in I, C_{i}: X \rightarrow 2^{Y_{i}}$ is a set-valued mapping such that $C_{i}(x)$ is a closed pointed and convex cone with $\operatorname{int}_{i}(x) \neq \emptyset$ for each $x \in X$, and the set-valued mapping $M_{i}=Y_{i} \backslash\left(-\operatorname{int}_{i}\right): X \rightarrow 2^{Y_{i}}$ be upper semicontinuous;

(ii) for each $i \in I, T_{i}: X \rightarrow 2^{L\left(E_{i}, Y_{i}\right)}$ is an upper semicontinuous set-valued mapping with nonempty compact values, $\eta_{i}: X_{i} \times X_{i} \longrightarrow E_{i}$ be continuous with respect to the second argument;

(iii) for each $i \in I$, there exists a mapping $h_{i}: X_{i} \times X_{i} \rightarrow Y_{i}$, such that:

(a) For all $x=\left(x^{i}, x_{i}\right) \in X, \forall y_{i} \in X_{i}, \exists v_{i} \in T_{i}(x)$, such that $h_{i}\left(x_{i}, y_{i}\right)$ $-\left\langle v_{i}, \eta_{i}\left(y_{i}, x_{i}\right)\right\rangle \in-\operatorname{intC}_{i}(x)$

(b) For all $x=\left(x^{i}, x_{i}\right) \in X$, the set $\left\{y_{i} \in X_{i}: h_{i}\left(x_{i}, y_{i}\right) \in-\operatorname{intC}_{i}(x)\right\}$ is convex;

(c) For all $x=\left(x^{i}, x_{i}\right) \in X, h_{i}\left(x_{i}, x_{i}\right) \notin-\operatorname{intC}_{i}(x)$.

Proof: It is only needed to show that (b) of (iii) in Theorem 3.4 holds. If the condition (b) of (iii) in Theorem 3.4 does not hold, then there exists a finite set $\left\{y_{i_{1}}, y_{i_{2}}, \ldots, y_{i_{n}}\right\}$ $\subseteq X_{i}$ and some point $x=\left(x^{i}, x_{i}\right) \in X$ with $x_{i}=\sum_{j=1}^{n} \alpha_{j} y_{i_{j}}$, where $\alpha_{j} \geqslant 0$ and $\sum_{j=1}^{n} \alpha_{j}=1$, satisfying $h_{i}\left(x_{i}, y_{i_{j}}\right) \in-\operatorname{intC}_{i}(x)$ for all $j \in\{1,2, \ldots, n\}$. that is, $y_{i_{j}} \in\left\{y_{i} \in X_{i}\right.$ : $\left.h_{i}\left(x_{i}, y_{i}\right) \in-\operatorname{intC}_{i}(x)\right\}$ for all $j \in\{1,2, \ldots, n\}$. By the convexity of the set $\left\{y_{i}\right.$ $\left.\in X_{i}: h_{i}\left(x_{i}, y_{i}\right) \in-\operatorname{intC}(x)\right\}$, we have $x_{i} \in\left\{y_{i} \in X_{i}: h_{i}\left(x_{i}, y_{i}\right) \in-\operatorname{intC}_{i}(x)\right\}$. Hence, $h_{i}\left(x_{i}, x_{i}\right) \in-\operatorname{intC_{i}}(x)$, which contradicts to the condition (c) of (iii). Then, by Theorem 3.4, we know that the conclusion holds.

REMARK 3.2. By the results in section 3, it is easy to obtain the existence results for all of the special models of the system of generalised set-valued quasi-variationallike inequalities mentioned in the section 2. For example, let $I=\{1\}$, by Theorem 3.1, Corollary 3.2, Theorem 3.4 and Corollary 3.5, respectively, we obtain the existence 
results of a solution for generalised set-valued quasi-variational-like inequalities which are generalisations of the main results in [21] and Theorem 1 in [31] from the cases of generalised set-valued variational-like inequalities to the cases of generalised set-valued quasi-variational-like inequalities.

\section{REFERENCES}

[1] E. Allevi, A. Gnudi and I.V. Konnov, 'Generalized vector variational inequalies over product sets', Nonlinear Anal. 47 (2001), 573-582.

[2] Q.H. Ansari, 'A note on generalized vector variational-like inequalities', Optimization 41 (1997), 197-205.

[3] Q.H. Ansari, 'Extended generalized vector variational-like inequalities for nonmonotone multivalued maps', Ann. Sci. Math. Quebec 21 (1997), 1-11.

[4] Q.H. Ansari, S. Schaible and J.C. Yao, 'Systems of vector equilibrium problems and its applications', J. Optim. Theory Appl. 107 (2000), 547-557.

[5] Q. H. Ansari and J.C. Yao, 'A fixed-point theorem and its applications to the systems of variational inequalities', Bull. Austral. Math. Soc. 59 (1999), 433-442.

[6] J.P. Aubin and I. Ekeland, Applied nonlinear analysis (J. Wiley and Sons, New York, 1984).

[7] M. Bianchi, Pseudo P-monotone operators and variational inequalities, Report 6 Istituto di econometria e Matematica per le decisioni economiche (Universita Cattolica del Sacro Cuore, Milan, Italy, 1993).

[8] D. Chan and J.S. Pang, 'The generalized quasi-variational inequality problem', Math. Oper. Res. 7 (1982), 211-222.

[9] G.Y. Chen, 'Existence of solutions for a vector variational inequality: an existension of the Hartmann-Stampacchia theorem', J. Optim. Theory Appl. 74 (1992), 445-456.

[10] G.Y. Chen and G.M. Cheng, 'Vector variational inequalities and vector optimization', Lecture Notes in Econom. and Math. Systems 285 (1987), 408-456.

[11] G.Y. Chen and B.D. Craven, 'Approximate dual and approximate vetor variational inequality for multiobjective optimization', J. Austral. Math. Soc. Ser. A 47 (1989), 418-423.

[12] G.Y. Chen and B.D. Craven, 'A vector variational inequality and optimization over an efficient set', Z. Oper. Res. 3 (1990), 1-12.

[13] G.Y. Chen and S.J. Li, 'Existence of solutions for a generalized vector variational inequality', J. Optim. Theory Appl. 90 (1996), 321-334.

[14] G.Y. Chen and X.Q. Yang, 'Vector complementarity problems and its equivalences with the weak minimal element in ordered spaces', J. Math. Anal. Appl. 53 (1990), 136-158.

[15] G. Cohen and F. Chaplais, 'Nested monotony for variational inequalities over a product of spaces and convergence of iterative algorithms', J. Optim. Theory Appl. 59 (1988), 360-390.

[16] A. Daniilidi and N. Hadjisavvas, 'Existence theorems for vector variational inequalities', Bull. Austral. Math. Soc. 54 (1996), 473-481.

[17] X.P. Ding, 'Existence of solutions for generalized quasi-variational-like inequalities', $A$ ppl. Math. Mech. 18 (1997), 141-150. 
[18] X.P. Ding, 'Generalized variational-like inequalities with nonmonotone set-valued mappings', J. Optim. Theory Appl. 95 (1997), 601-613.

[19] X.P. Ding, 'The generalized vector quasi-variational-like inequalities', Comput. Math. Appl. 37 (1999), 57-67.

[20] X.P. Ding and K.K. Tan, 'Generalized variational inequalities and generalized quasi-variational inequalities', J.Math. Anal. Appl. 148 (1990), 497-508.

[21] X.P. Ding and E. Tarafdar, 'Generalized vector variational-like inequalities without monotonicity', in Vector variational inequalities and vector equilibrium: mathematical theoreies, (F. Giannessi, Editor), Nonconvex Optim. Appl. 38 (Kluwer Academic Publishers, Dordrecht, 2000), pp. 113-123.

[22] X.P. Ding and E. Tarafdar, 'Generalized vector variational-like inequalities with $C_{x}-\eta$-pseudomonotone set-valued mappings', in Vector variational inequalities and vector equilibrium: mathematical theoreies, (F. Giannessi, Editor), Nonconvex Optim. Appl. 38 (Kluwer Academic Publishers, Dordrecht, 2000), pp. 125-140.

[23] K. Fan, 'Fixed -point and minimax theorems in locally convex topological linear spaces', Proc. Nat. Acad. Sci. U.S.A. 38 (1952), 121-126.

[24] F. Giannessi, 'Theorems alternative, quadratic programs, and complementarity problems', in variational inequalities and complementarity problems, (R.W. Cottle, F. Giannessi, and J.L. Lions, Editors) (John Wiley and Sons, New york, 1980), pp. 151-186.

[25] J. Kelley and I. Namioka, Linear topological space (Springer-Verlag, New York, Heidelberg, Berlin, 1963).

[26] I.V. Konnov and J.C. Yao, 'On the generalized vector variational inequality problem', $J$. Math. Anal. Appl. 206 (1997), 42-58.

[27] G.M. Lee, D.S. Kim and S.J. Cho, 'Generalized vector variational inequality and fuzzy extension', Appl. Math. Lett. 6 (1993), 47-51.

[28] G.M. Lee, D.S. Kim and B.S. Lee, 'Generalized vector variational inequality', Appl. Math. Lett. 9 (1996), 39-42.

[29] G.M. Lee, B.S. Lee and S.S. Chang, 'On vector quasivariational inequalities', J. Math. Anal. Appl. 203 (1996), 626-639.

[30] K.L. Lin, D.P. Yang and J.C. Yao, 'Generalized vector variational inequalities', J. Optim. Theory Appl. 92 (1997), 117-125.

[31] Q. Luo, 'Generalized vector variational-like inequalities', in Vector variational inequalities and vector equilibrium: mathematical theoreies, (F. Giannessi, Editor), Nonconvex Optim. Appl. 38 (Kluwer Academic Publishers,, Dordrecht, 2000), pp. 363-369.

[32] E. Michael, 'A note on paracompact spaces', Proc. Amer. Math. Soc. 4 (1953), 831-838.

[33] W. Oettli and D. Schlager, 'Existence of equilibria for monotone multivalued mappings', Math. Methods Oper. Res. 48 (1998), 219-228.

[34] J.S. Pang, 'Asymmetric variational inequality problems over product sets: Applications and iterative methods', Math. Programming 31 (1985), 206-219.

[35] H.H. Schaefer, Topological vector space (Springer-Verlag, New York, 1971).

[36] M.H. Shih and K.K. Tan, 'Generalized quasi-variational inequalities in locally convex topological vector spaces', J. Math. Anal. Appl. 108 (1985), 333-343.

[37] A.H. Siddiqi, A.H. Ansari and A. Khaliq, 'On vector variational inequality', J. Optim. Theory Appl. 84 (1995), 171-180. 
[38] C.H. Su and X.Z. Yuan, 'Some fixed point theorems for condensing multifunctions in locally convex spaces', Proc. Nat. Acad. Sci. U.S.A. 50 (1975), 150-154.

[39] G.Q. Tian and J.X. Zhou, 'Quasi-variational inequalities without the concavity assumption', J. Math. Anal. Appl. 132 (1993), 289-299.

[40] X.Q. Yang, 'Vector complementarity and minimal element problems', J. Optim. Theory Appl. 77 (1993), 483-495.

[41] X.Q. Yang, 'Vector variational inequality and duality', Nonlinear Anal. 21 (1993), 869-877.

[42] X.Q. Yang, 'Generalized convex functions and vector variational inequality', J. Optim. Theory Appl. 79 (1993), 563-580.

[43] X.Q. Yang and J.C. Yao, 'Gap functions and existence of solutions to set-valued vector variational inequalies', J. Optim. Theory Appl. 115 (2002), 407-417.

[44] S.J. Yu and J.C. Yao, 'On vector variational inequality', J. Optim. Theory Appl. 89 (1996), 749-769.

[45] X.Z. Yuan and E. Tarafdar, 'Generalized quasivariational inequalities and some applications', Nonlinear Anal. 29 (1997), 27-40.

[46] J.X. Zhou and G. Chen, 'Diagonal convexity conditions for problems in convex analysis and quasi-variational inequalities', J. Math. Anal. Appl. 132 (1988), 213-225.

Department of Mathematics

Chongqing Normal University

Chongqing 400047

People's Republic of China
Department of Mathematics Inner Mongolia University

Hohhot 010021

Inner Mongolia

People's Republic of China

e-mail: pjw7503_cn@sina.com. 